\title{
Swarm Intelligence based Sparrow Search Optimization Algorithm for Node Localization in Wireless Sensor Networks
}

\author{
SANTHOSH. $\mathbf{M}^{1}$, SUDHAKAR. $\mathbf{P}^{2}$ \\ ${ }^{1}$ Research Scholar,Department of Computer Science and Engineering,FEAT, Annamalai \\ University,Annamalainagar - 608 002, santhosh395@gmail.com \\ ${ }^{2}$ Associate Professor,Department of Computer Science and Engineering, FEAT,Annamalai \\ University,Annamalainagar - 608 002, kar.sudha@gmail.com
}

\begin{abstract}
In recent times, wireless sensor networks (WSNs) gained significant attention among researchers owing to its applicability in several domains. Node localization is considered as a major design issue in WSN, which has the ability to estimate the location of the sensor nodes by the use of anchor nodes. It can be considered as an optimization problem and swarm intelligence (SI) algorithms can be employed to resolve it. This paper presents a sparrow search optimization algorithm based node localization technique called SSOA-NL for WSN. The presented SSOA-NL model is based on the group wisdom, foraging and anti-predation behavior of sparrows. Initially, the sensor nodes undergo random deployment and node initialization process takes place. Then, every node executes the SSOA-NL technique to locate itself by determining the coordinate points and become localized nodes. At the next round, the localized nodes will be appended to the set of anchor nodes to carry out the further localization process. The performance of the SSOA-NL technique has been assessed under diverse dimensions and a comprehensive comparative results analysis takes place. The experimental results ensured that the SSOA-NL technique is found to be superior to other methods.
\end{abstract}

Key words: Node localization, WSN, Sparrow Search Algorithm, Anchor nodes, Swarm intelligence

\section{INTRODUCTION}

In general, wireless sensor network (WSN) is well-known and novel technology which offers effective benefits in diverse applications such as medical sector, surveillance, astronomy, armed forces, farming and so on. WSN is composed of extensive prospects because of its robust, simple development, as well as self-organization. Also, WSN contains massive count of small, cheaper independent sensor nodes which may be homogenous or heterogeneous for the purpose of monitoring climatic conditions [1]. These independent nodes are useful for sensing, processing and convey the collected data from an atmosphere through Wireless Ad Hoc Network (WANET) to Base Station (BS) or sink node that is considered as a last destination. The architecture of WSN is depicted in figure 1. The diverse optical, biological, chemical, magnetic sensors have been included for the nodes for calculating external factors. The characteristic of WSN such as self-organization and instantaneous development makes promising results in WSN applications. The evaluation of the location of the sensor nodes is considered as the challenging issues of WSN and named as localization problem.

The process of node is to place and observe nodes, where the observed data are useful, in which data collected from BS would be insignificant for an individual with no localization data of nodes in a sensor field. A localization is determined as evaluating the place of unknown sensor nodes named as destination nodes with the application of well-known location of sensor nodes termed as anchor nodes on the basis of measurement like time variations in arrival, angle of arrival, triangulation, higher likelihood and so on. The localization problem of WSN could be solved under the application of Global Positioning System (GPS) with a sensor node; however it is not applicable because of limited energy, maximized expense and size problems. Moreover, it cannot be applied for indoor and underwater. Hence, effective and best alternative is essential for placing the sensor nodes.

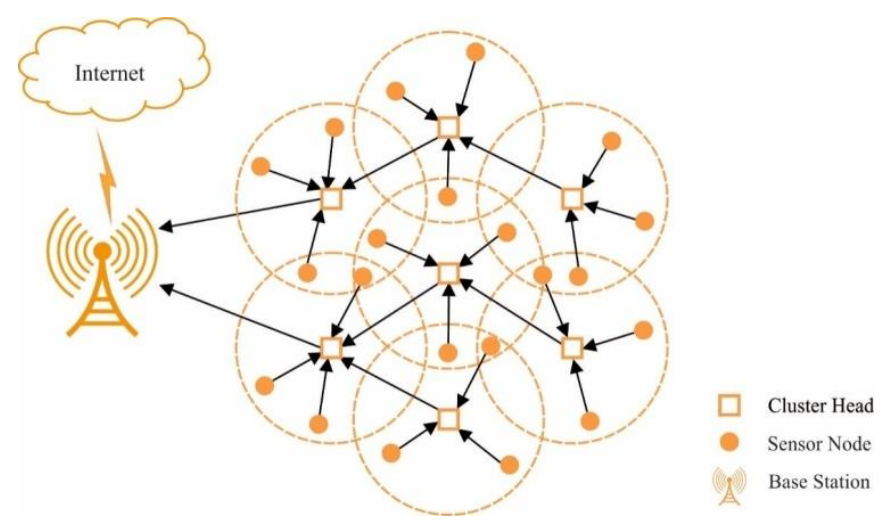

Figure 1: Structure of WSN 
Diverse non-GPS-based localization methods are applied and classified as range-based as well as range-free methodologies. Here, location is determined using trilateration of anchor nodes. Range-free localization does not need range details among target node as well as anchor node; thus, it is derived from topological data. Furthermore, technologies offer maximum accuracy than range-free localization methods, however it is cheaper. The range-based localization of sensors is accomplished using 2 stages namely, Ranging and Position evaluation stages. At the initial phase, every target node calculates the distance from anchor nodes under the application of intensity of obtained signal time. Accurate distance estimation is impossible due to the presence of noise. Secondly, the position of sensor nodes is evaluated under the application of data collected from ranging phase. It is operated by applying geometric model or optimization scheme.

In past decades, various interesting models were applied for resolving the issues of WSN node localization. In [2], a brief study of several localization systems for ubiquitous processing has been projected. In [3], distinct localization models with brief study of numerous measurement models to WSNs are defined. In [4], a new localization method where anchor nodes flood the location of target nodes in the system. Moreover, target node determines the place by exploiting position of massive anchor nodes. Moreover, in [5], an extended version of similar method is projected, where target node applies the neighbors' position for enhancing the position accuracy. In [6], Kalman filter-based least-square evaluation mechanism is applied for reporting the problem of error accumulation. In [7], convex optimization-relied on semi-definite programming has been applied to report the node localization issues in WSN.

In [8], semi-definite programming technique is expanded to non-convex inequality limitations and for gradient-search, correspondingly. The optimization methods are optimized for resolving optimization issues such as decision subset sum issues, Feature Selection (FS), travelling salesman problem. Localization problem is assumed as unconstrained optimization issues applied with optimization methodologies. The explanatory models of optimization are ineffective in resolving localization problem due to the time and complexity aspects. It has inspired the developers to apply efficient and effective nature-based meta-heuristic approaches for resolving the issue. Such models are evolved in resolving different optimization issues by maintaining the appropriate balance between these elements. The methods like Genetic Algorithm (GA), Particle Swarm Optimization (PSO), and Firefly Algorithm (FA) were applied for placing the sensor nodes. There are diverse optimization methodologies that are utilized for reducing localization error and localize the massive target nodes. In Sharma and Kumar [9], developers have presented a distributed range-free node localization models for 3D WSNs according to the GA. Likewise, in Peng and $\mathrm{Li}$ [10], by using localization model which applies GA, localization accuracy of unwanted nodes in WSNs was enhanced. In addition, a new range free localization method depends upon GA and connectivity has been projected. The SI model which is provided in the study was PSO. PSO meta-heuristics performs the searching operation by accelerating flocks of birds and fish schooling behaviors. This meta-heuristics are utilized extensively for node localization in WSNs.

This paper develops a sparrow search optimization algorithm (SSOA) based node localization technique called SSOA-NL for WSN. The presented SSOA-NL model makes use of the group wisdom, foraging and anti-predation behavior of sparrows to perform node localization process. Firstly, the sensor nodes undergo random deployment and node initialization process takes place. Afterwards, every node executes the SSOA-NL technique to locate itself by determining the coordinate points and become localized nodes. At the next round, the localized nodes will be appended to the set of anchor nodes to carry out the further localization process. In order to validate the performance of the SSOA-NL technique, a series of simulations takes place under diverse anchor nodes. The attained simulation results ensured the goodness of the SSOA-NL model over the compared methods.

\section{THE PROPOSED SSOA-NL TECHNIQUE}

The working principle of the proposed SSOA-NL technique is depicted in figure. 2. As depicted, the sensor nodes undergo random deployment and form a network. Then, the nodes perform initialization process to gather details regarding its neighbors. Afterwards, each node executes the SSOA-NL technique to localize itself and determined its coordinate points. At the next round, the localized nodes will be appended to the set of anchor nodes to carry out the further localization process.

\subsection{Nature of sparrows}

Usually, sparrows are gregarious birds. It exists in massive portion of the world and desires to live in places where the human also resides. Unlike the other models, alternate small birds, the sparrow is highly effective and contains efficient storage. It is apparent that, there are 2 diverse kinds of captive house sparrows, the producer as well as scrounger. The producers highly explore the food source, whereas scroungers get the food from producers. Moreover, the witness showcases that the birds normally apply behavioral principles like flexibly, and switch among producers and scrounging.

Moreover, the food source can be identified by using the strategy of producer and the scrounger. The works have depicted that the individuals observe the behaviour of the other members in the group. Simultaneously, the intruders in the bird flock that has to enhance the predation rate are applied for computing the food resources of companions with higher intakes. Moreover, the energy preservation of 
individuals plays a significant role if the sparrow selects diverse foraging principles, and sparrow's minimum energy preserves scrounge in maximum number. It is highly applicable and it is placed on the periphery of population, which can be attacked by predators and prominently attempts to reach best place. It is apparent that the animals, that is placed on the centre, which moves nearby neighbours and reduce the risk of an application. For sample, if a bird predicts a predator, maximum individual's chirps and whole group moves.

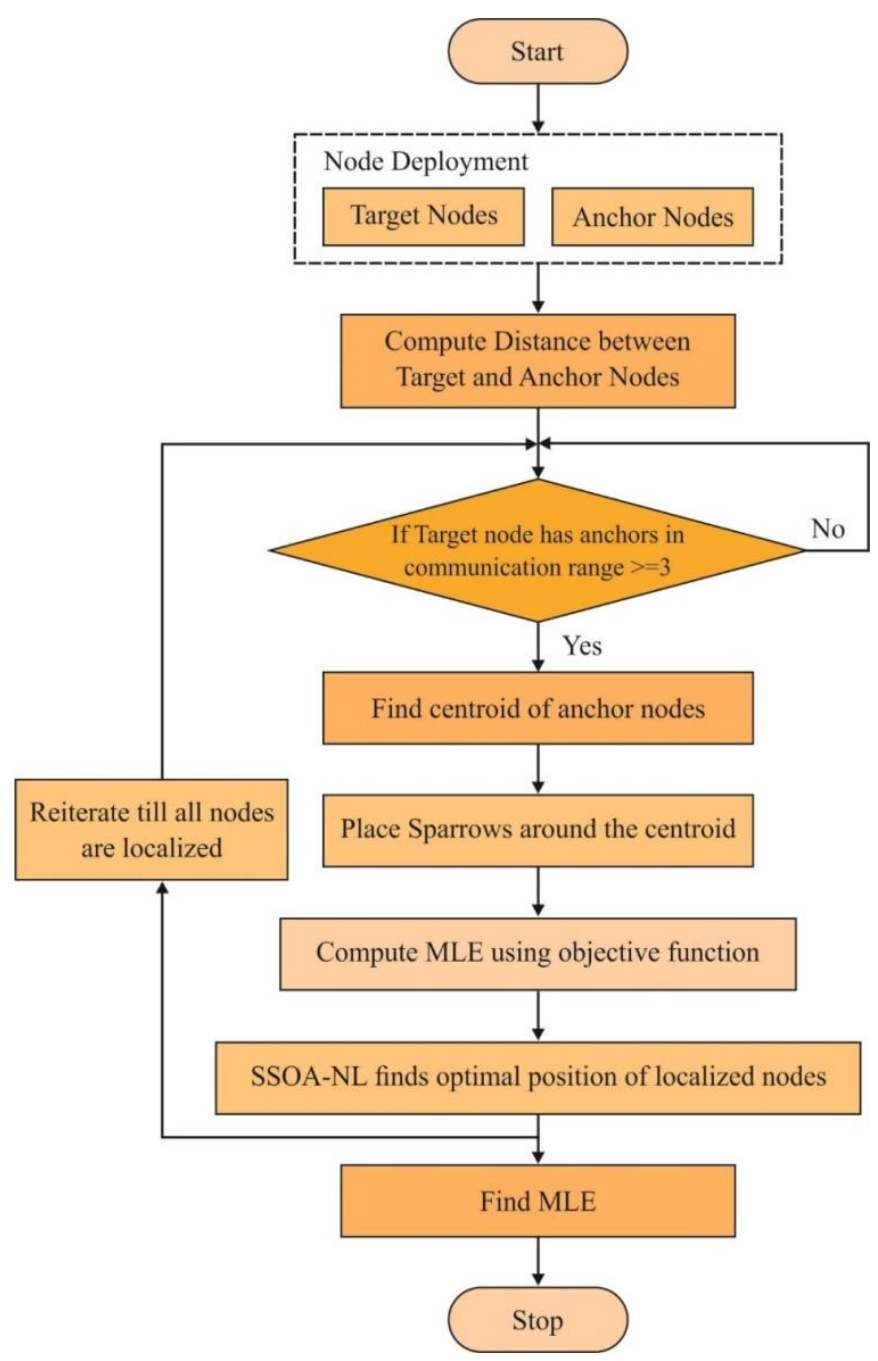

Figure 2: Overall Process of Proposed Model

\subsection{SSOA for Node Localization Process}

The node localization in WSN involves the determination of the coordinate points of the higher number of unknown nodes on the basis of a lower number of anchor nodes under single-hop based range in the distributed WSN. The major steps of node localization methods are:

i. $\quad N$ beacons nodes as well as $M$ unknown nodes are placed in random manner in the WSN, associated with similar transmission radius as $N$ and $M$.

ii. The beacon nodes estimate the locations and forward the coordinates according to RSSI for estimating position of unknown nodes.

iii. Every node which is encircled by maximum anchors is assumed as localized node.

iv. Every node which are localized estimates the distance $d_{j}$ from neighboring beacons by trilateration $(N=3)$ or multilateration $(N>3)$. An actual distance among the target node $(x, y)$ and $\mathrm{i} \square$ th anchor $\left(x_{j}, y_{i}\right)$ which is defined as given below:

$$
d_{i}=\sqrt{\left(x-x_{i}\right)^{2}+\left(y-y_{i}\right)^{2}} .
$$

The distance values are influenced by various paths and obstacles would block them by considering the climatic situation, that is implied as noise $n_{i}$. The determined distance $\hat{d}_{i}$ among the target node $(x, y)$, and $\mathrm{i} \square$ th anchor $\left(x_{i}, y_{i}\right)$ which is labeled by the function:

$$
\hat{d}_{i}=\left[d_{i}+n_{i}\right] .
$$

- Every node which are localized and runs the SSOA-NL technique in an independent manner for placing themselves by estimating adjacent coordinates $(x, y)$. The objective function is determined by the given expression:

$$
f(x, y)=\frac{1}{N} \sum_{i=1}^{N} \sqrt{\left(x-x_{i}\right)^{2}+\left(y-y_{i}\right)^{2}}-\hat{d}_{i},
$$

where $N \geq 3$ denotes the count of beacon nodes with communication radius.

- The nodes which are developed at the final iteration would be included to collection of nodes which is treated as anchor nodes in upcoming iteration.

- The steps 2-6 are followed until satisfying the termination criteria. The mean localization error (MLE) is determined as mean square of Euclidean distance for evaluated node coordinates $\left(x_{i}, y_{i}\right)$ as well as real node coordinates; $\left(X_{i}, Y_{i}\right)$ for $i=$ $1,2, \ldots M L$ is computed for IBAT with the help of given function:

$$
E_{L}=\frac{1}{M_{L}} \sum_{i=1}^{L} \sqrt{\left(x_{i}-X_{i}\right)^{2}+\left(y_{i}-Y_{i}\right)^{2}},
$$

where $L=M-N$," $M$ denotes the count of localized nodes.

\section{PERFORMANCE VALIDATION}

The performance of the presented SSOA-NL technique has been evaluated under varying number of anchor nodes and transmission range. The presented SSOA-NL technique is simulated using MATAB tool. Figure 3 shows the sample node deployment with a set of localized and anchor nodes in the network. The parameter setting is given in Table 1. 
Table 1: Parameter settings

\begin{tabular}{|l|l|}
\hline Parameters & Values \\
\hline Node count & 300 \\
\hline Area & $200 * 200\left(\mathrm{~m}^{2}\right)$ \\
\hline Anchor nodes & $10-100$ \\
\hline Transmission Range & $10-40 \mathrm{~m}$ \\
\hline Iteration count & 100 \\
\hline
\end{tabular}

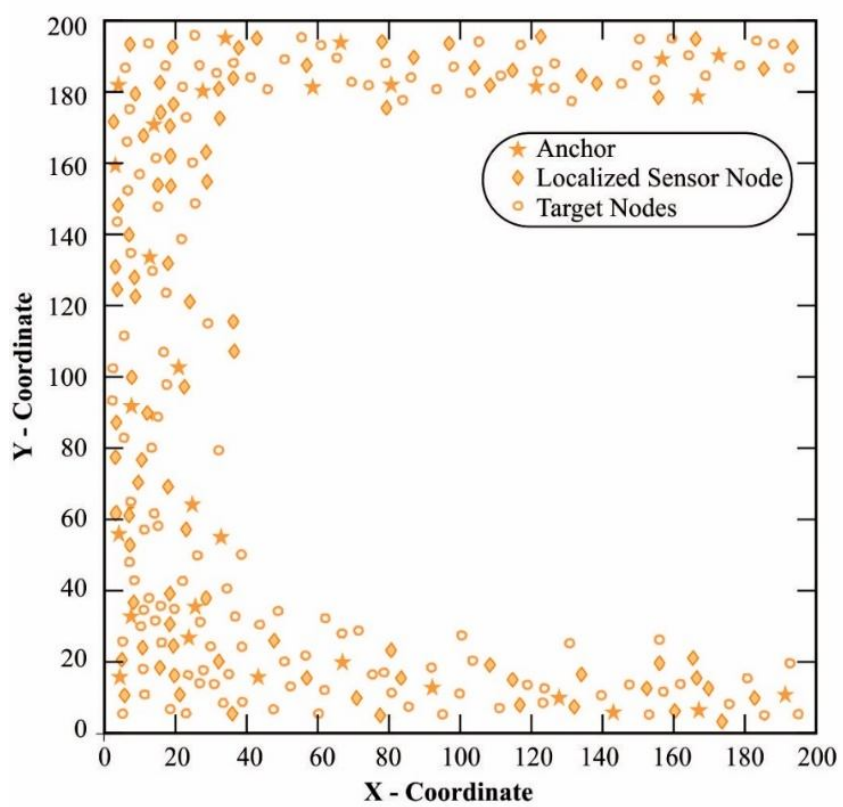

Figure 3: Node Localization using SSOA

\subsection{Results Analysis interms of MLE}

Figure 4 provides a detailed comparative analysis of the SSOA-NL technique interms of MLE under varying anchor node count. The figure has shown that the PSO algorithm has failed to localize the nodes effectively and reached to a maximum MLE. At the same time, the M-BAT algorithm has outperformed the PSO algorithm and attained slightly lower MLE. Similarly, the FOA algorithm has exhibited optimal performance over the PSO and M-BAT algorithms with an even lower MLE. Likewise, the GWO algorithm has demonstrated somewhat reasonable performance with a low MLE. Followed by, the BOA technique has tried to outperform all the compared methods, but the presented SSOA-NL technique has surpassed all the earlier methods by attaining a minimum MLE under all applied anchor nodes.

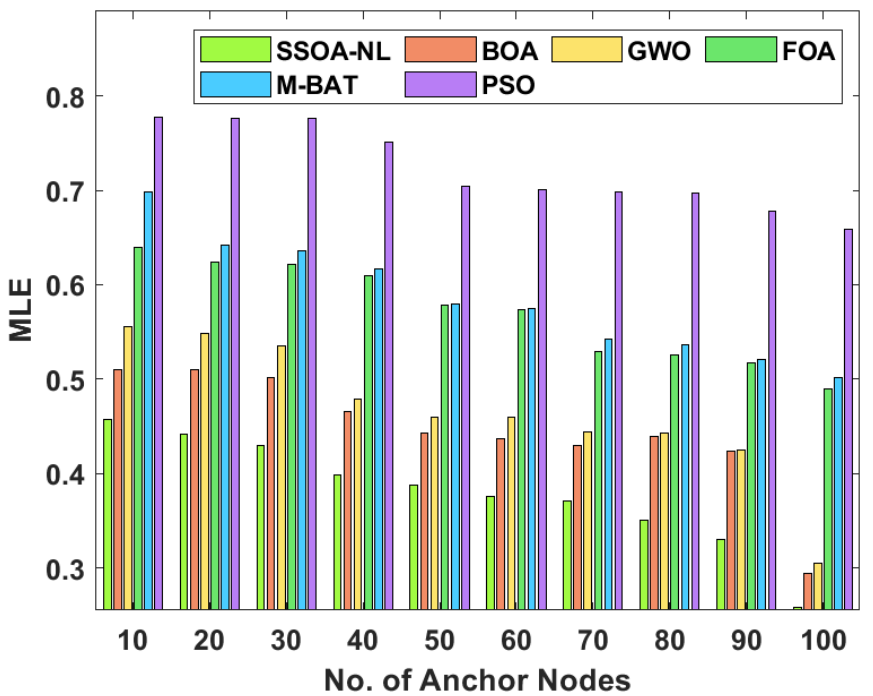

Figure 4: MLE analysis of SSOA-NL model

For instance, under the existence of 10 anchor nodes, the presented SSOA-NL technique has reached to a least MLE of 0.0457 whereas the BOA, GWO, FOA, M-BAT and PSO algorithms have resulted to a higher MLE of 0.511, 0.556, $0.640,0.698$ and 0.777. Similarly, under the presence of 50 anchor nodes, the SOOA-NL technique has achieved a minimal MLE of 0.388 whereas the BOA, GWO, FOA, M-BAT and PSO algorithms have resulted to a higher MLE of $0.443,0.459,0.579,0.580$ and 0.704 . Likewise, under the occurrence of 100 anchor nodes, a lower MLE of 0.258 has been achieved by SSOA-NL technique and the BOA, GWO, FOA, M-BAT and PSO algorithms have obtained a higher MLE of $0.294,0.305,0.489,0.502$ and 0.659 respectively.

\subsection{Results Analysis interms of localization time}

Figure 5 offers a brief comparative analysis of the SSOA-NL model with respect to localization time under various anchor node count. The figure has implied that the PSO method is not suitable to localize the nodes efficiently and attained a higher localization time. Simultaneously, the M-BAT approach has surpassed the PSO technology and reached better least localization time. Likewise, the FOA method has represented moderate performance over the PSO and M-BAT methodologies with uniform lower localization time. Similarly, the GWO scheme has depicted considerable performance with minimum localization time. Next, the BOA model has attempted to perform well than the earlier models; however the projected SSOA-NL approach has outperformed the compared schemes by accomplishing lower localization time under all applied anchor nodes. 


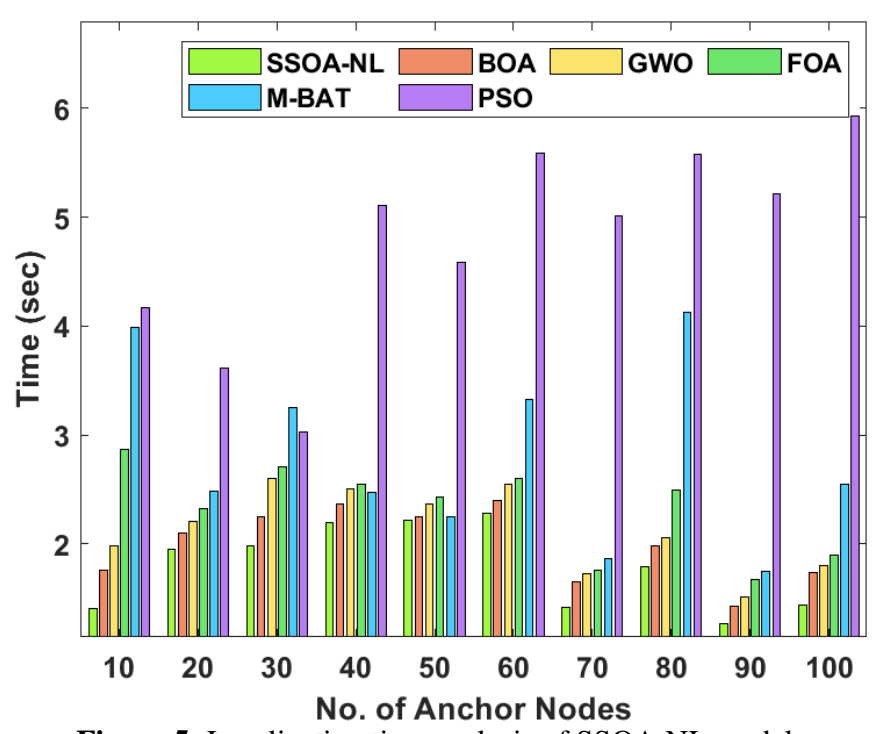

Figure 5: Localization time analysis of SSOA-NL model

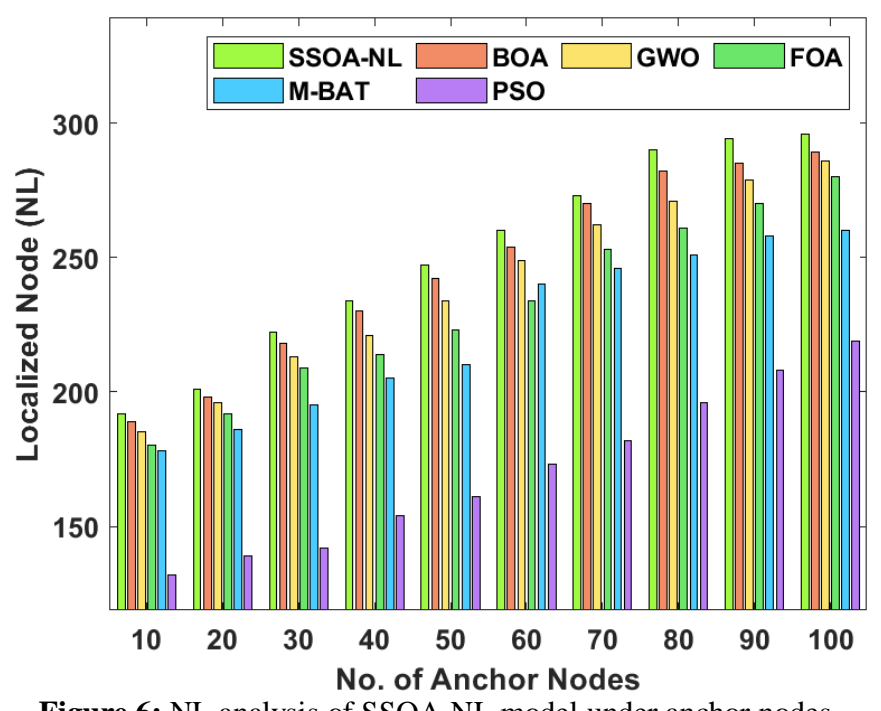

Figure 6: NL analysis of SSOA-NL model under anchor nodes

Figure 6compute the localization function of the SSOA-NL models with previous technologies under different anchor node count. The figure has implied that the PSO model has failed to localize the nodes productively and attained lower NL. Simultaneously, the M-BAT method has surpassed the PSO method and achieved moderate NL. Likewise, the FOA framework has represented considerable performance over the PSO and M-BAT models with acceptable NL. In line with this, the GWO approach has depicted better performance with a higher NL. Next, BOA model has attempted to perform well than existing approaches; however the projected SSOA-NL scheme has outperformed the previous technologies by accomplishing higher NL under all applied anchor nodes.

\section{CONCLUSION}

This paper develops a sparrow search optimization algorithm (SSOA) based node localization technique called SSOA-NL for WSN. The sensor nodes undergo random deployment and form a network. Then, the nodes perform initialization process to gather details regarding its neighbors. Afterwards, each node executes the SSOA-NL technique to localize itself and determined its coordinate points. Subsequently, the localized nodes will be appended to the set of anchor nodes to perform the further localization process. These processes get iterated till all the unknown nodes in the network are localized. A detailed experimentation takes place and the results are assessed interms of MLE, NL and computation time. The outcome of the experiments depicted that the SSOA-NL technique has attained optimal node localization related to other methods. In future, the energy efficient clustering techniques can be applied to improve the network lifetime of WSN.

\section{REFERENCES}

1. Akyildiz, I.F.; Su, W.; Sankarasubramaniam, Y.; Cayirci, E.: Wireless sensor networks: a survey. Comput. Netw. 38(4), 393-422 (2002)

2. Hightower, J.; Borriello, G.: Location systems for ubiquitous computing. Computer 8, 57-66 (2001)

3. Mao, G.; Fidan, B.; Anderson, B.D.:Wireless sensor network localization techniques. Comput. Netw. 51(10), 2529-2553 (2007)

4. Chandra Sekhar Reddy, N. Purna Chandra Rao Vemuri, Govardhan, A. An Emperical Study on Support Vector Machines for Intrusion Detection, International Journal of Emerging Trends in Engineering Research, Vol. 7, No. 10, pp. 383-387, October 2019.

5. Rufo I. Marasigan Jr, Alvin SarragaAlon, Mon Arjay F. Malbog, Joshua S. Gulmatico, Copra Meat Classification using Convolutional Neural Network,International Journal of Emerging Trends in Engineering Research, Vol. 8. No. 2, February 2020

6. Subramaniyam, S, Performance Analysis on Diesel Engine using Neem and Soya Bean Oil,International Journal of Emerging Technologies in Engineering Research (IJETER), Vol. 5, Issue 8, August 2017.

7. Savvides, A.; Park, H.; Srivastava, M.B.: The n-hop multilateration primitive for node localization problems. Mob. Netw. Appl. 8(4), 443-451 (2003)

8. Doherty, L.; Pister, K.S.; El Ghaoui, L.: Convex position estimation in wireless sensor networks. In: INFOCOM 2001. Twentieth Annual Joint Conference of the IEEE Computer and Communications Societies. Proceedings, vol. 3, pp. 1655-1663 (2001)

9. Biswas, P.; Lian, T.-C.; Wang, T.-C.; Ye, Y.: Semidefinite programming based algorithms for sensor network localization. ACM Trans. Sens. Netw. (TOSN) 2(2), 188-220 (2006)

10. Sharma, G.; Kumar, A. Improved range-free localization for three-dimensional wireless sensor networks using genetic algorithm. Comput. Electr. Eng. 2018, 72, 808-827.

11. Peng, B.; Li, L. An improved localization algorithm based on genetic algorithm in wireless sensor networks. Cognit. Neurodyn. 2015, 9, 249-256. 